\title{
SURFACE TENSION, STICKINESS AND ENGULFMENT
}

\author{
R. H. Ottewill, A. B. Schofield and J. A. Waters \\ School of Chemistry, University of Bristol, \\ Cantock's Close, Bristol, BS8 1TS, UK
}

\begin{abstract}
The thermodynamic conditions for the engulfment of one set of particles by another has been given in terms of interfacial energies. Experimentally, it has been shown that a polymer with a high glass transition temperature can be engulfed by a particle of low glass transition temperature; also, that inorganic particles can be engulfed by polymer particles. As a precursor to then engulfment stage heterocoagulation can be used for bringing the particles together in a "sticking" mode. This appears to be a general process which is applicable to a number of scientific areas, e.g. in biology, phagocytosis, and in material science for the preparation of composite particles.
\end{abstract}

\section{INTRODUCTION}

The mechanism of engulfment of one particle by another has been one of long-standing interest in many areas of research. For example in physiology, phagocytosis is the process whereby the white cells or leucocytes in the blood remove invading foreign bodies by engulfment and ingestion. In 1927 it was recognised by Ponder [1,2] that there were three important factors to consider in the mechanism of phagocytosis; these were surface tension, stickiness and

Present address:

A.B. Schofield, Dept. of Physics \& Astronomy, University of Edinburgh, Edinburgh, EH9 3JZ, U.K.

J.A. Waters, Dept. of Chemical Engineering, Imperial College of Science, London, U.K. 
engulfment. However, he also mentioned that:- " .. unfortunately, stickiness is a property which although perfectly familiar, is not capable of being satisfactorily defined in exactness .. ". An excellent review of current knowledge in 1934 was published by Mudd et al [3]. Almost at the same time the ideal that a physical attraction existed between colloidal particles was proposed which led to the classical paper by Hamaker [4] on the force of attraction between two spherical colloidal particles. Subsequently the Hamaker Constant was introduced relating the nature of the particles and the medium to the magnitude of the attraction. This with the later introduction of the theories of colloid stability by Derjaguin and Landau [5] and Verwey and Overbeck [6] linked the stickiness of particles to ideas of colloid stability. This was examined in the context of phagocytosis by Wilkins et al $[7,8]$. The work of Good and Girifalco [9] and Fowkes [10] then linked the Hamaker Constant to the dispersion contribution to surface tension. Van Oss et al in 1973 [11] extended these ideas to give a correlation between Hamaker Constants and phagocytosis and various ideas about the polar contributions to surface energy $[12,13,14]$.

In a recent series of papers ideas relating to the engulfment of particles have been developed in the area of Polymer Colloids in terms of surface tension, using it in a predictive sense $[15,16,17,18]$. Then both the engulfment and encapsulation were successfully demonstrated experimentally $[15,19]$. Heterocoagulation was used to provide a mechanism of sticking small particles to a larger host particle. Control of this process then led to engulfment of one particle by another or alternatively to spreading of smaller particles on a larger one to form a surface layer by encapsulation. This brought together on a " quantitative " basis the earlier quantitative ideas of the importance of surface tension, stickiness and ingestion ( engulfment).

It is clear, however, that these ideas have a much wider applicability and in this paper as a tribute to the fundamental work carried out by Carel Jan van Oss in this area we summarise the concepts of engulfment. We also demonstrate, as examples, the 


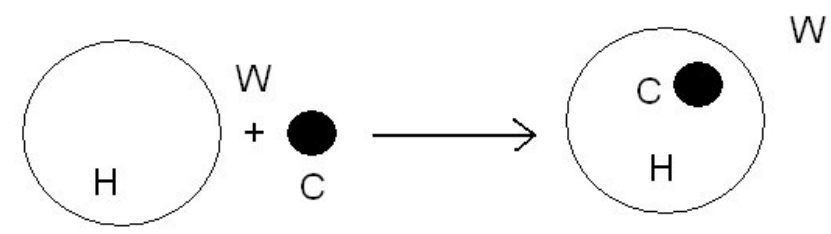

Fig. 1 Schematic representation of the engulfment of a Core Particle, $\mathrm{C}$ by a Host particle, $\mathrm{H}$, in water, W.

engulfment of polystyrene particles and magnetic iron oxide particles into particles of polybutylmethacrylate

\section{THEORY}

In previous work [15] in the area of polymer colloids it has been shown that the conditions for the engulfment of a core particle $\mathrm{C}$ of volume $\mathrm{V}_{\mathrm{C}}$ into a host particle $\mathrm{H}$ of volume $\mathrm{V}_{\mathrm{H}}$, as illustrated in Figure 1, are given by:

$$
\left(\gamma_{C W}-\gamma_{C H}\right) / \gamma_{H W}>\left(1-\phi_{H}^{2 / 3}\right) / \phi_{C}^{2 / 3}
$$

in an aqueous medium, W. In equation (1) $\gamma_{\mathrm{CW}}=$ the interfacial energy of the core-water interface; $\gamma_{\mathrm{CH}}=$ the interfacial energy of the corehost interface and $\gamma_{\mathrm{HW}}=$ the interfacial energy at the host-water interface. Also, $\phi_{\mathrm{H}}=$ the volume fraction of the host particles and $\phi_{\mathrm{C}}=$ the volume fraction of the core particles, such that $\phi_{\mathrm{H}}+\phi_{\mathrm{C}}$ $=1.0$ where $\phi_{\mathrm{H}}=\mathrm{V}_{\mathrm{B}} /\left(\mathrm{V}_{\mathrm{H}}+\mathrm{V}_{\mathrm{C}}\right)$ and $\phi_{\mathrm{C}}=\mathrm{V}_{\mathrm{C}} /\left(\mathrm{V}_{\mathrm{C}}+\mathrm{V}_{\mathrm{H}}\right)$.

The left hand side of equation (1) is the interfacial energy expression in the form of Young's equation for a droplet of $\mathrm{H}$ on a planar substrate $\mathrm{C}$ in an aqueous medium $\mathrm{W}$, namely,

$$
\left(\gamma_{C H}-\gamma_{C W}\right) / \gamma_{H W}=\cos \theta
$$

where $\theta$ is the contact angle. Complete spreading of the host over the core can be achieved when $\cos \theta=1.0$. However, kinetic factors also have to be considered and internal re-arrangement of the host particle morphology towards the thermodynamically favoured structure may be completely inhibited or be very slow depending on the internal 
and interfacial viscosities of the host particle. For example, in the case of polymer colloidal particles if the host particle is cross-linked or in the glassy state particle transfer by engulfment can be either very slow or completely inhibited.

The interfacial energy expressions in equations (1) and (2) can be increased to values of unity or greater by:-

i) increasing $\gamma_{\mathrm{CW}}$ by making the core particles more hydrophobic,

ii) decreasing $\gamma_{\mathrm{HW}}$ by making the host particle more hydrophilic,

iii) decreasing $\gamma_{\mathrm{CW}}$ by making the surface of the core particle more like that of the host particle.

Hence, full particle engulfment can occur for $\left(\gamma_{\mathrm{CW}}-\gamma_{\mathrm{CH}}\right) / \gamma_{\mathrm{HW}}$ either equal to or greater than 1.0 providing that the kinetic factors are favourable.

\section{EXPERIMENTAL}

Polystyrene (PS) Particles

These were prepared as a latex by an emulsifier-free polymerisation [20] of styrene at $70^{\circ} \mathrm{C}$ using ammonium persulphate as the initiator, as previously described $[15,19]$. The particles so prepared were anionic in character over the $\mathrm{pH}$ range utilised.

Polybutylmethacrylate (PBMA) Particles

Emulsifier-free polymerisation at $70^{\circ} \mathrm{C}$ was used with $2.2^{\prime}$ azobis2-amidinopropane dihydrochloride as the initiator to prepare a PBMA latex. This procedure which has been described previously [22] produced particles which were cationic, as shown by electrophoresis, below $\mathrm{pH}$ 8. However, by grafting methoxy-polyethyleneglycol methacrylate to the particle surfaces, during the last stages of the polymerisation, particles were formed which were colloidally stabilised both electrostatically and sterically [22].

\section{Magnetite Particles}

A dispersion of magnetite particles was prepared using the procedure described by Massart[23]. Initially $10 \mathrm{~cm}^{3}$ of $2.0 \mathrm{~mol} \mathrm{dm}{ }^{-3}$ 
ferrous chloride in $2.0 \mathrm{~mol} \mathrm{dm}^{-3}$ hydrochloric acid was mixed with 40 $\mathrm{cm}^{3}$ of $1.0 \mathrm{~mol} \mathrm{dm}{ }^{-3}$ ferric chloride. The mixture was then poured into $500 \mathrm{~cm}^{3}$ of $0.7 \mathrm{~mol} \mathrm{dm}{ }^{-3}$ ammonium hydroxide. The black precipitate formed was allowed to settle after which the supernatant was removed. The remaining dispersion was then dialysed against an ammonium hydroxide-ammonium chloride buffer solution at $\mathrm{pH} 8.25$ for 10 days, with changes of the dialysate solution every 24 hours.

\section{RESULTS}

Stickiness

As a precursor to the process of engulfment it is necessary to cause the smaller particle, the one to be engulfed, to stick to the host particle. As one method of providing a sticking mechanism we have made use of the natural surface charge of the particles by adjusting the $\mathrm{pH}$ of the solution so that one particle was anionic and the other cationic at this $\mathrm{pH}$ thus leading to heterocoagulation by a combination of electrostatic and van der Waals' attraction [18]. Other methods can be used to achieve this effect such as, the addition of a surfactant of opposite charge in order to produce a low surface charge on one type of particle [16].

Engulfment of PS particles by PBMA particles

The particles sizes of the PBMA and PS particles were determined by transmission electron microscopy (Figures $2 a$ and $2 b$ ) and the glass transition temperature, $\mathrm{T}_{\mathrm{g}}$, by differential scanning calorimetry. These data are given in TABLE 1 .

For the heterocoagulation stage small aliquots of the two latices were mixed in a small sample bottle and then vigorously shaken. Prior to the mixing process the volume fraction and number concentration of each latex was determined from the w/w concentration.

The heterocoagulated units produced consisted of the larger PBMA particles with the smaller PS particles adsorbed on to the surface, as illustrated in Figure 2c. The concentration of PS particles was chosen arbitrarily to be that needed to cover one-quarter of the 


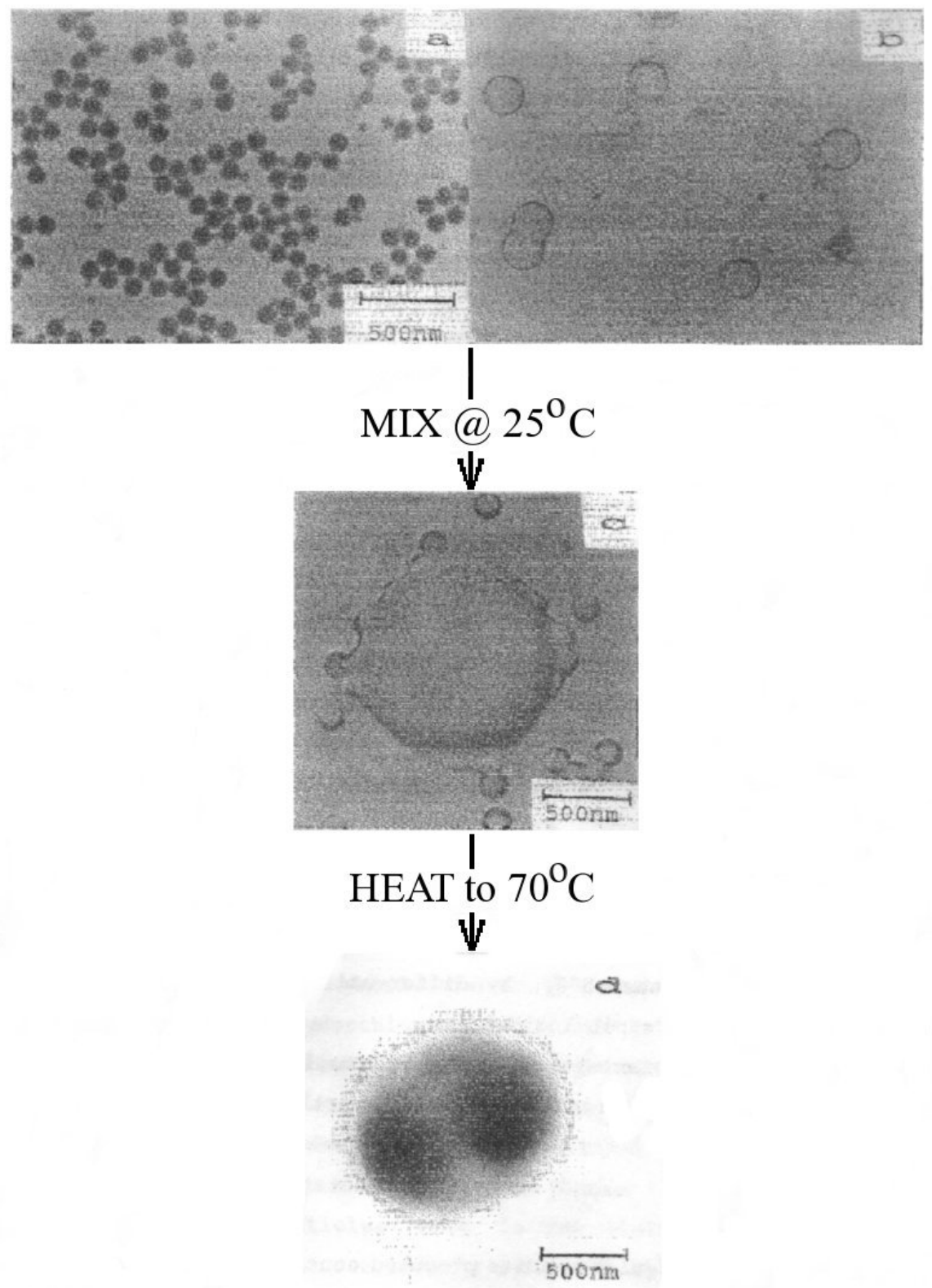

Fig 2 Transmission electron micrographs of:- a) anionic PS particles, radius $45 \mathrm{~nm}$ and $\mathrm{T}_{\mathrm{g}} 106^{\circ} \mathrm{C}$; b) cationic PBMA particles, radius $446 \mathrm{~nm}$ and $\mathrm{T}_{\mathrm{g}} 21^{\circ} \mathrm{C}-27^{\circ} \mathrm{C}$; c) heterocoagulated unit (carbon replica); d) engulfed PS particles in a PBMA particle 
TABLE 1

Engulfment of PS by PBMA

$\begin{array}{ccccc}\text { Role } & \text { Polymer } & \text { Radius } / \mathrm{nm}^{+} & \mathrm{T}_{\mathrm{g}}{ }^{\circ} \mathrm{C} & \text { Charge } \\ \text { HOST } & \text { PBMA } & 446 \pm 14 & 25 \pm 4 & + \\ \text { CORE } & \text { PS } & 45 \pm 13 & 106 \pm 2 & - \\ & & & \end{array}$

surface area of the PBMA particles assuming that complete adsorption occurred. During the addition process the electrolyte concentration was maintained at $10^{-5} \mathrm{~mol} \mathrm{dm}{ }^{-3}$ sodium chloride and a $\mathrm{pH}$ of ca. 4 . After standing for $24 \mathrm{~h}$ at room temperature the mixed system was ultrasonicated for 30 minutes to create a good dispersion of the coated PBMA particles.

In order to achieve engulfment of the PS particles into the PBMA particles the temperature was raised to $70^{\circ} \mathrm{C}$ and maintained at this temperature for $24 \mathrm{~h}$. This temperature was ca. $45^{\circ} \mathrm{C}$ above the $\mathrm{T}_{\mathrm{g}}$ of PBMA and hence these particles were in the rubbery state. As can be seen from figure $2 \mathrm{~d}$ under these conditions engulfment of the PS particles occurred. Since PS gives a more electron dense image in transmission microscopy than PBMA the PS particles are directly discernable as the blacker units in the micrograph.

Engulfment of Inorganic Particles by PBMA

Magnetite was chosen as an example of an inorganic colloid for engulfment into a polymer particle on the basis that it would produce composite magnetic particles. The magnetite particles produced are illustrated by the transmission electron micrograph shown in figure 3. The radius of the particles produced was $16.5 \pm 3 \mathrm{~nm}$. Well cleaned magnetite particles are reported [21] to have an isoelectric point at $\mathrm{pH}$ 7. This provided a window between $\mathrm{pH} 7$ and $\mathrm{pH} 8$ where the inorganic particles were negatively charged and the PBMA particles were positively charged.

For these experiments the PBMA particles were freshly prepared and cleaned. The number average particles radius was $145 \pm 14 \mathrm{~nm}$ and 


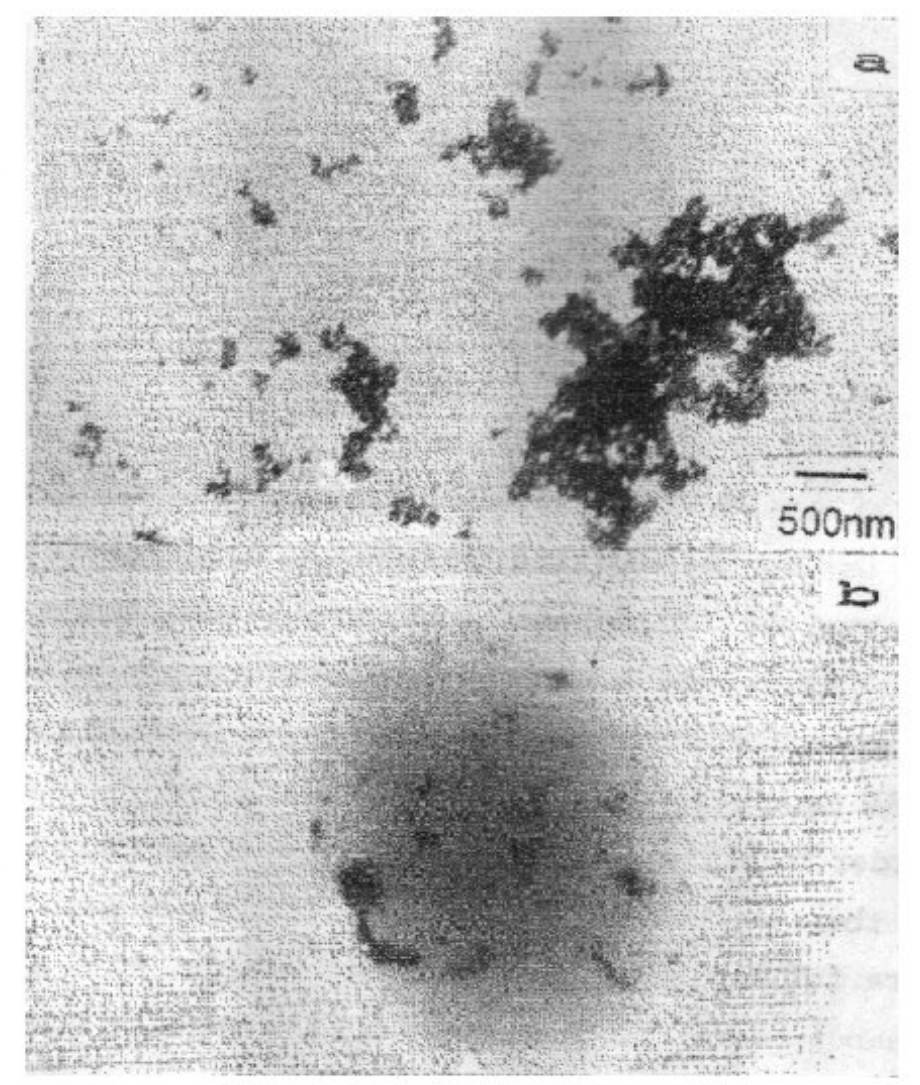

Fig 3 Transmission electron micrographs of:- a) magnetite Particles; b) magnetite particles engulfed in a PBMA particle

the isoelectric point of the particles was $\mathrm{pH}$ 8.0. Previous experience with these particles has shown that on aging slow hydrolysis of the amidine groups can occur leading to a lowering of the isoelectric point owing to a loss of positive charge $[13,16,18]$; also these particles can be sensitive to impurities leached from the container.

Mixing of magnetite and PBMA particles was carried out in a similar manner to that described earlier except that the $\mathrm{pH}$ was controlled at $\mathrm{pH}$ 7.5. Figure 3 shows transmission electron micrographs of the magnetite particles and also a micrograph of a 


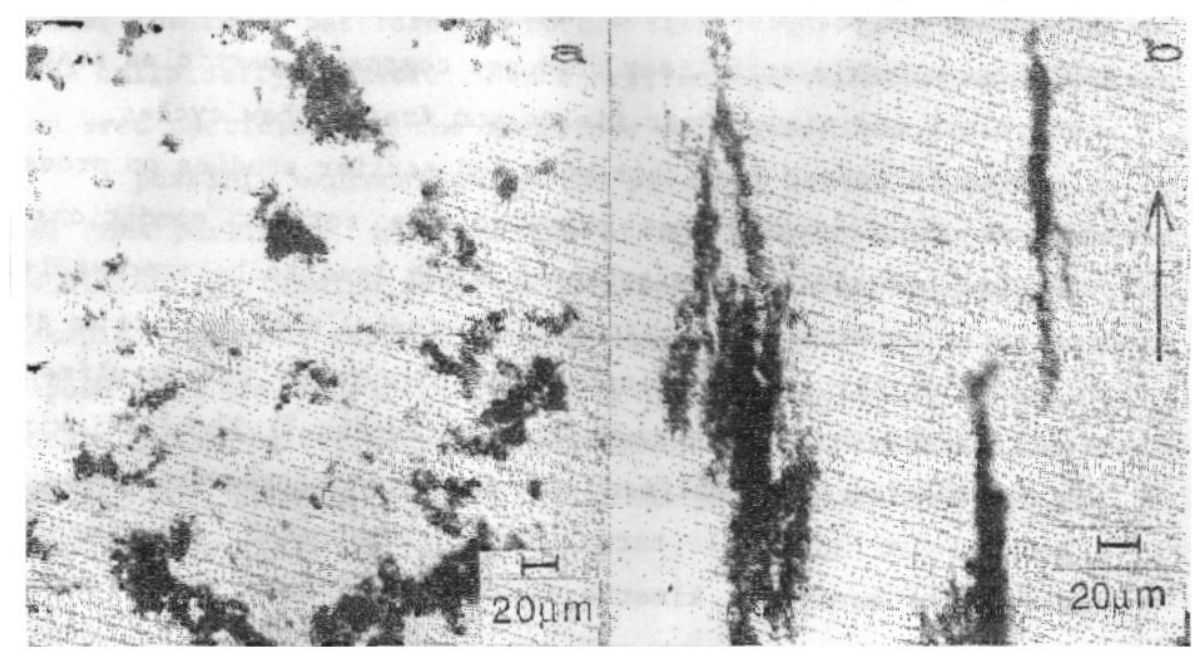

Fig 4 Optical micrographs of an aqueous dispersion of PBMA-magnetite particles; a) in the absence and b) in the presence of a magnetic field $\uparrow$, direction of the magnetic field

PBMA particle with engulfed magnetite particles; engulfment was also confirmed by electron microscopy of thin sections cut from the particles.

The magnetic properties of these particles were demonstrated by examining a sample using an optical microscope in the absence and presence of a magnetic field. The results are shown in figure 4 and clearly indicate alignment of the composite particles in the direction of the field.

\section{DISCUSSION}

The principles discussed in the theory section and in previous papers [15-19] have been used to examine experimentally the engulfment of polymer particles with a high $\mathrm{T}_{\mathrm{g}}$ into particles with a low $\mathrm{T}_{\mathrm{g}}$. Similarly, it has been possible to engulf inorganic particles, magnetite, into PBMA particles with a low $\mathrm{T}_{\mathrm{g}}$. Inorganic particles can also be encapsulated by a similar process [25]. The use 
of methoxy-polyethyleneglycol methacrylate ( molecular weight ca. 2000 ) as a steric stabiliser produced composite particles stable to electrolyte addition, shear fields and freeze-thaw cycles.

It would appear from this work and earlier studies on processes such as phagocytosis that provided the correct conditions for colloidal interactions are achieved which lead to heterocoagulation ( particle sticking ) and that the interfacial energies are as stated in equation (1) then conditions are favourable for engulfment to occur i.e the principles enunciated by Ponder [1,2] of stickiness, surface tension and engulfment are paramount. Moreover, they can be expressed in physical terms and demonstrated experimentally. However, as mentioned kinetic factors also have to be carefully considered.

This would suggest that the engulfment process may well be more general than often recognised and one which can occur in a number of systems. One example, is the synthesis of copolymer particles by emulsion or emulsifier-free polymerisation. An example of this was found in the previous work of Lye [26] where a seed latex of polymethyl methacrylate (PMMA), radius $176 \mathrm{~nm}$ was used as a seed in a polymerisation reaction with styrene. With the seed particles in water at $70^{\circ} \mathrm{C}$ styrene was added and then after 15 minutes sodium persulphate was added to initiate polymerisation of the styrene monomer. The particles radius after polymerising for $24 \mathrm{~h}$ increased to $193 \mathrm{~nm}$. No secondary formation of particles was observed and differential scanning calorimetry showed that two polymer phases were present in the final particles. An electron microscope examination of thin sections of the particles showed small discrete particles of polystyrene around the periphery of the final copolymer particle. This effect was confirmed by extraction of the particles using cyclohexane and making a re-examination by electron microscopy. The micrographs clearly revealed small spherical cavities around the external surface of the particles; apparently where the polystyrene units had been removed.

Moreover, in a previous investigation [27] evidence was obtained which indicated that following nucleation of particles in the aqueous 
phase, the small particles so formed became swollen with monomer and were colloidally unstable. Hence heterocoagulation occurred between the seed particles and the small new particles.

A possible explanation of the presence of the PS particles in the PMMA particles, in the light of the present work, is that the conditions pertaining were those likely to favour engulfment. For example, with the surface swollen with styrene after a short exposure to the monomer followed by heterocoagulation of the newly formed small particles engulfment seems highly probable and would explain the frequently found " currant bun " particles observed in copolymer particle synthesis.

\section{ACKNOWLEDGMENTS}

We wish to thank ICI Paints Division for support of this work from the Strategic Research Fund and also Dr Neil Williams of the Division for a number of useful discussions. One of us (RHO) also thanks the DTI Colloid Technology Programme for support during the period of this work

\section{REFERENCES}

1. Ponder, E., Protoplasm $\underline{3}$, 611, (1927)

2. Ponder E., J. Gen. Physiol. 11, 757, (1927)

3. Mudd, E., McCutcheon, M. and Lucke, Physiol.

Rev. 14, 210, (1934)

4. Hamaker, H. C., Physica, 4, 1058 (1937)

5. Derjaguin, B. V. and Landau, L., Acta Physicochem.

U.R.S.S. 14, 633 (1941)

6. Verwey, E. J. W. and Overbeck, J. Th. G., Theory of

Stability of Lyophobic Colloids, Elseveir,

Amsterdam

7. Wilkins, D. J., Ottewill, R. H., and Bangham A. D.,

J. Theoret. Biol., 2, 165 \& 176 (1962)

8. Wilkins, D. J., Ph. D. Thesis, University of

Cambridge (1960)

9. Good, R. J. and Girifalco, L. A., J. Phys. Chem.

64, 561 (1960) 
10. $\quad$ Fowkes, F., Ind. Eng. Chem. 56, 40 (1964)

11. Neumann, A. W., van Oss, C. J. and Szekely, J., Kolloid Z. u. Z. Polymere, 251, 415 (1973)

12. Neumann, A. W., Omenyi, S. N. and van Oss, C. J., Colloid Polym. Sci., 257, 413 (1979)

13. van Oss, C. J., Chaudhury M. K. and Good, R. J., Chem. Rev., 요, 927 (1988)

14. van Oss, C. J., Interfacial Forces in Aqueous Media, M. Dekker, New York (1994)

15. Ottewill, R. H., Schofield, A. B. and Waters, J. A., Colloid Polym. Sci., 274, 763 (1996)

16. Waters, J.A., US Patent 5210113 (1990); European Patent 0327199 (1993); US Patent 5296524 (1993)

17. Waters, J.A. Colloids and Surfaces, $\underline{83}, 167$, (1994)

18. Waters, J. A., in Goodwin, J. W. and Buscall R. (eds.), Colloidal Polymer Particles, Academic Press, London, (1995)

19. Ottewill, R. H., Schofield, A. B., Waters, J. A. And Williams, N. St. J., Colloid Polym. Sci., 275, 274, (1997)

20. Goodwin, J. W., Hearn, J., Ho, C. C. and Ottewill, R. H., Colloid Polym. Sci., 252, 464, (1974)

21. Goodwin, J. W., Ottewill, R. H. and Pelton, R. Colloid Polym. Sci., 257, 61, (1979)

22. Ottewill, R. H. and Satgurunathan, R., Colloid Polym. Sci., 273, 379, (1995)

23. Massart, R., IEEE Trans. Mag. $\underline{17}$, 1247, (1981)

24. Dixon, D. R., Water Res. 18, 529, (1984)

25. Ottewill, R. H., Schofield, A. B. and Waters, J. A. to be published

26. Lye J. Ph. D. thesis, University of Bristol (1987)

27. Chung-Li, Y., Goodwin, J. W. and Ottewill, R. H., Prog. Colloid Polym. Sci., 60, 163 (1976) 